\title{
Philosophiques
}

\section{Le monopole confessionnaliste dans son rapport avec la réflexion sur l'éthique}

\section{Louise Marcil-Lacoste}

Volume 4, numéro 1, avril 1977

URI : https://id.erudit.org/iderudit/203068ar

DOI : https://doi.org/10.7202/203068ar

Aller au sommaire du numéro

Éditeur(s)

Société de philosophie du Québec

ISSN

0316-2923 (imprimé)

1492-1391 (numérique)

Découvrir la revue

Citer ce document

Marcil-Lacoste, L. (1977). 4. Le monopole confessionnaliste dans son rapport avec la réflexion sur l'éthique. Philosophiques, 4(1), 125-136.

https://doi.org/10.7202/203068ar d'utilisation que vous pouvez consulter en ligne.

https://apropos.erudit.org/fr/usagers/politique-dutilisation/ 


\title{
4. LE MONOPOLE CONFESSIONNALISTE DANS SON RAPPORT AVEC LA RÉFLEXION SUR L'ÉTHIQUE.
}

\author{
par Louise Marcil-Lacoste
}

Pour clarifier le sens de mon commentaire, il est nécessaire de considérer le contexte où se pose la question des fondements de la morale et de son enseignement au Québec. Concrètement, ce que l'on appelle ici "l'enseignement de la morale " se situe dans le contexte précis suivant : certains parents (pour le niveau élémentaire) et certains étudiants (pour le niveau secondaire) demandent d'être exemptés de l'enseignement religieux et moral confessionnel qui est obligatoire dans les écoles. En accédant à cette demande d'exemption, on doit offrir un "enseignement de la morale " en substitut de l'enseignement moral et religieux confessionnel. En parlant

2. H.A. Hodges, Wilbelm Diltbey, London, 1944, p. 19.

3. Cité par A. Stein, Der Begriff des Verstehens bei Dilthey, p. 55. 
de l'enseignement de "la morale ", on ne parle donc pas de l'enseignement de la morale inspirée de la confessionnalité catholique ou protestante. On parle d'un certain programme qui sera le substitut de cette dernière. On parle également, ceci est important pour comprendre la suite de mon propos, d'une morale-substitut qui, pour des raisons de cohérence et de ce que l'on appelle le respect de la majorité, ne doit pas contredire l'enseignement moral et religieux confessionnel.

D'une manière générale, à propos de ce contexte, je dis que dans le système scolaire public québécois des niveaux élémentaire et secondaire, l'enseignement de la morale apparaît comme le moyen (insatisfaisant du reste) par lequel on tente d'atténuer les effets du monopole confessionnaliste en matière de programmes d'enseignement.

En considérant le problème philosophique des fondements de la morale sous l'angle précis et déterminé de ce que cette expression désigne dans le système public québécois - une morale "substitut de " et " compatible avec " la morale confessionnelle - je donne à ma question un enjeu spécifiable et concret. Et c'est dans le contexte de cette référence que j'essaie de voir comment les communications ici reproduites permettent de faire avancer le débat. Or, ce qui me frappe à ce sujet, c'est leur commune incapacité d'élucider les problèmes que pose le monopole confessionnaliste, partant leur commune tendance à justifier le statu quo, soit directement, soit par défaut. J'entends : il est difficile de dire pourquoi il faudrait changer quelque chose d'important au monopole confessionnaliste en partant des principes énoncés par les panélistes, soit parce qu'ils s'y rattachent et l'épaulent, soit parce qu'ils le critiquent en défendant les principes même sur lesquels le monopole confessionnaliste repose.

Bien entendu, il serait futile de reprocher aux participants au panel consacré aux fondements de l'enseignement de la morale de n'avoir pas résolu un problème qu'ils ne se sont pas donné pour objet de traiter ou qu'ils n'ont pas voulu aborder en priorité. De même, il serait carrément grotesque d'invoquer le silence des panélistes sur une question, en l'occurrence le monopole confessionna. liste, pour conclure aux intentions mystificatrices, approbatrices ou désapprobatrices des intervenants en en ce qui le concerne. Cela du reste n'est pas mon propos. 
Les circonstances de mon intervention sont les suivantes: dans le débat qui suivit la présentation des communications ici reproduites, on aborda, entre autres, la question de savoir pourquoi finalement on enseigne la morale. $\hat{A}$ cette question, j'ai alors soutenu qu'il y avait au Québec une réponse politique qui réside dans l'atténuation des effets du monopole confessionnaliste. On m'a ensuite demandé de donner les raisons pour lesquelles je tenais cette réponse politique pour une réponse ayant une portée philosophique. C'est ce que je vais tenter de faire ici. J'essaierai de montrer comment la reconstruction des enjeux philosophiques inhérents à l'enseignement de la morale à partir d'une analyse contextuelle permet de détecter à quel point la réflexion philosophique reproduit à grands traits une problématique déterminée d'avance par le monopole confessionnaliste. Cette reconstruction vise à mettre en lumière les raisons pour lesquelles à différents moments d'un débat animé, j'avais néanmoins l'impression qu'à un certain niveau, il n'y avait pas de "désaccord fondamental " entre les panélistes ${ }^{1}$.

Ma thèse est donc philosophiquement la suivante : une partie essentielle des enjeux inhérents à l'enseignement de la morale et aux problèmes des fondements auxquels la justification de cet enseignement renvoie doit être posée explicitement à partir d'une analyse contextuelle. On peut théoriquement étayer cette assertion de la double façon suivante : (1) l'historicité même des théories et des pratiques éthiques est indéterminée en l'absence de mises en perspective contextuelles; (2) la valeur d'une réflexion métaéthique dans le domaine de l'enseignement de la morale relève de sa capacité d'éclairer les rapports complexes entre abstraction et concrétude, modèle et contexte, formalisme et contenu. Mais pour les fins de ce commentaire, il sera suffisant de faire remarquer qu'en l'absence d'une analyse explicite du contexte québécois dans lequel se pose le problème de l'enseignement de la morale, on en vient à proposer des réflexions qui ont plus l'allure d'une photographie (philosophique) des problèmes vécus contextuellement que l'allure de réflexions par lesquelles il serait possible de faire avancer le débat au point où il se trouve en ce moment. J'ajoute immédiatement que je nie pas l'élément d'éclairage que procure

1. Puisque j'effectue ici une reconstruction, on comprendra que je ne discuterai pas des thèses en présence en elles-mêmes mais bien du point de vue de leurs rapports les unes aux autres, compte tenu de la question que je pose. 
une "photographie ". Je tiens qu'il est important de s'apercevoir que l'on en fait une, quand cela est le cas.

Voici donc, rapidement, du reste, quelques éléments d'une image globale du contexte où se pose notre problème.

\section{LA MORALE NATURELLE}

Comme je l'ai dit, l'enseignement de la morale apparaît dans le système scolaire public québécois dans le contexte des exemptions aux cours obligatoires d'enseignement moral et religieux de type confessionnel, catholique ou protestant. Comme je l'ai également signalé, l'enseignement de la morale aux exemptés (peu nombreux du reste, les pourcentages variant entre trois et huit pour cent) doit être effectué à partir d'un programme (qui n'existe pas encore au plan provincial), lequel programme doit être compatible avec le programme d'enseignement confessionnel.

I1 n'est pas nécessaire d'épiloguer sur les raisons pour lesquelles c'est précisément la morale que l'on appelle "la morale naturelle " qui semble appropriée dans la recherche d'un programme compatible avec le projet chrétien. Depuis le Moyen-Âge, on connaît l'importance des thèses relatives à la morale naturelle ou à la loi naturelle pour assurer la démonstration du caractère rationnel de la théologie catholique ainsi que les rapports entre la raison et la foi. On connaît aussi l'importance de cette notion de morale naturelle dans l'histoire des théologies chrétiennes en général.

Sur le plan spécifique d'un programme d'enseignement de la "morale naturelle ", il est donc relativement aisé de concevoir la compatibilité d'un programme de morale naturelle et d'un programme de morale chrétienne. Il suffit, en quelque sorte, d'amputer un ensemble de notions de leur spécificité théologique et de leur justification théologique. Par exemple, il est relativement aisé de produire une liste de vertus comme la prudence, la justice, la force et la tempérance, ce qui est vrai du reste des "vertus théologales " transposées, par exemple, en "vision du monde "pour la foi, en "morale de l'engagement " pour l'espérance, et en solidarité ou fratenité pour la charité. De ce dernier point de vue, il semblerait bien que le fardeau de la preuve de la spécificité repose plutôt sur les épaules des théologiens que sur celles des " naturalistes ". Mais apparemment, peu de gens s'intéressent aujourd'hui 
à l'élaboration des preuves en vertu desquelles telle ou telle valeur serait dite "catholique " plutôt que "presbytérienne " ou " anglicane ", ou tout simplement " humaine ", la morale naturelle étant ce par quoi les valeurs et principes sont présentés privativement, c'est-à-dire sans avoir à recourir à des notions ou à des justifications de type confessionnel. Cela cependant, et par voie de retour, permet de penser que les morales confessionnelles chrétiennes sont en réalité des morales naturelles, pour peu qu'on les allège de leur justification théologique. Cette thèse est justement celle qu'il faut maintenir si l'on veut défendre le monopole confessionnaliste.

Première remarque critique : aborder les problèmes philosophiques relatifs à l'enseignement de la morale sous l'angle prioritaire de la morale naturelle (en pour ou contre), c'est aborder les problèmes de la morale sous l'angle sous lequel il est nécessaire de les aborder en situation de monopole confessionnaliste. Plusieurs "fuites en avant " sont ici possibles. Par exemple, on pourra dire que puisque la morale naturelle ne saurait être donnée comme une morale absolue, elle ne saurait être enseignée ou en tout cas elle ne saurait être enseignée comme si elle était un absolu. Raisonner ainsi. c'est raisonner très exactement comme la plupart des confessionnalistes, soit ceux qui posent à l'enseignement valable une condition absolue, soit ceux qui admettent facilement (cessons de les voir comme s'ils étaient inconscients des épistémologies contemporaines) le caractère relatif des formes de la vie et de la pensée morales. Du reste, de longue tradition, les confessionnalistes et les "naturalistes" savent fort bien que nul n'est parfait ; comme ils admettent, de moins longue tradition peut-être, qu'il faut enseigner que même l'Église est humaine.

Une autre "fuite en avant ", tout à fait typique à mon avis, est la suivante: étant donné que toute tentative de fonder la morale et d'en justifier l'enseignement est fatalement marquée du sceau de la relativité, renonçons à cette démarche et bornons-nous à montrer les limites inéluctables qu'elle comporte chaque fois qu'elle sera reprise par quelqu'un d'autre. À beaucoup d'égards mais évidemment pas à tous -, raisonner ainsi c'est raisonner dans la forme même de la pensée confessionnaliste pour qui, au niveau principal, une morale relative est effectivement une contradiction dans les termes. C'est encore une fois le "tout ou rien" 
de principe. Ici, le contessionnaliste prendra le parti du tout, quitte à le vider de contenu en cours d'argumentation. Le relativiste prendra le parti non pas du rien, mais des "non-touts " successifs.

Il y a donc rapport étroit et, à vrai dire, institutionnellement nécessaire entre le discours sur la morale et le discours sur la confessionnalité chrétienne, ce lien étant assumé par la médiation de la notion de morale naturelle. Et je dis qu'en faisant de cette question l'enjeu principal du débat (en pour ou contre), on obéit à une problématique déterminée d'avance par le monopole confessionnaliste.

\section{L.EFORMALISHE}

Ayant posé ce rapport morale-confessionnalité, il n'est sûrement pas nécessaire de s'attarder sur le fait que le système scolaire public du Québec pour les niveaux élémentaire et secondaire est un système confessionnel. Sauf peut-être pour rappeler ici - la confusion est tellement fréquente - que nous n'avons toujours pas d'écoles neutres au Québec ni de commissions scolaires neutres?, bien que nous ayons des écoles et des commissions scolaires confessionnelles dont la plupart nous diront (l'Association des parents catholiques en tête) qu'elles sont "pratiquement" neutres. Si l'on veut, le sens dans lequel on peut et doit parler de nos institutions d'enseignement des niveaux élémentaire et secondaire comme étant dans une situation de monopole confessionnaliste est caractérisé par le formalisme.

Dites à un confessionnaliste que les écoles briment la liberté religieuse, il vous répondra que l'école est publique. Dites à un non-confessionnaliste que les écoles sont publiques, et il vous répondra qu'elles sont confessionnelles. En réalité, tout le monde a raison. Nos écoles sont toutes et confessionnelles et publiques non seulement dans leur statut juridique, mais également dans la conception des programmes d'enseignement en en particulier d'enseignement moral. De plus, comme pour ajouter à la confusion, nos écoles sont confessionnelles "de commune renommée ", ce qui signifie qu'elles sont toutes confessionnelles dans la mesure précise

2. Sauf peut être une commission scolaire ou quelques écoles dont on cite périodiquement l'existence en de tels débats. 
de leur attachement et de leur indifférence au projet confessionnel lui-même.

Dénième remarque critique : aborder les problèmes philosophiques de l'enseignement de la morale sous l'angle formel des problèmes posés par tout projet éthique, c'est aborder le problème sous l'angle rendu nécessaire par le monopole confessionnaliste, lequel se caractérise par son formalisme et notamment son formalisme juridique. Annoncer comme une nouvelle qu'il y a du pluralisme dans la société québécoise, la vieille unanimité en matières de morale et de religion étant rompue, et centrer son intervention au plan formel des problèmes de l'impossible universalité et de l'impossible unanimité en matières éthiques, c'est très exactement reproduire (adéquatement du reste) les raisons pour lesquelles le monopole confessionnaliste est devenu et a dû devenir formel. À mon avis cependant, ce n'est pas faire avancer le débat. De ce point de vue, seule une démarche qui aborderait les contenus éthiques aurait une valeur de rupture avec le formalisme inscrit dans la situation elle-même. À moins, bien entendu, de conclure que de l'éthique, on ne peut parler. Auquel cas, on laisse aussi "les choses comme elles sont ", y inclus le débat dont je parle."

\section{L.ABSENCE DE FONDEMENT}

Depuis la "révolution tranquille ", on assiste à une inversion des raisons motivant le maintien du caractère confessionnel des écoles et commissions scolaires et de la forme que prend la question de l'enseignement de la morale. Avant les années soixante, la confessionnalité du système scolaire correspondait dans ses grandes lignes à la confessionnalité de la plupart des institutions sociales québécoises. On justifiait alors le caractère confessionnel de l'École par sa valeur de reflet de l'attachement de l'ensemble des Québécois dans l'ensemble sociétal même - à l'Église du Québec. Depuis les années soixante, je parle ici surtout du secteur catholi-

Techniquement, considérer la confessionnalité comme étant "de commune renommée" consiste à reconnilître toutes les écoles et commissions scolaires comme confessionnelles à moins que ces dermieres ne demandent quion leur retire ce titre. Cette façon de décréter le caractère confessiomnel soppose à celle où les gens auraient à demander qu'on leur accorde ce statut.

j. Incidemment, on pourrait analyser la ressemblance entre cette dernière attitude méta-éthique et (clle. fort répandue qui consiste à dire qu'en réalité il n'y a pas de problème de confessionnalité au (Quélec. au sens oủ il est dit que ce problème est résolu ou se résoudra de lui-même par défiust. 
que, la confessionnalité scolaire apparaît comme marginale au plan des structures et institutions sociétales.

On n'a pas suffisamment remarqué que dorénavant, on justifie l'importance même du maintien de la confessionnalité des structures scolaires ainsi que du type d'enseignement moral qui s'y rattache par la marginalisation même des structures confessionnelles dans l'ensemble de la société québécoise. D'une manière générale en effet, on invoque la dissociation religion et vie pour justifier l'école confessionnelle. On dira, par exemple, que la pratique et la croyance religieuse d'une population n'influencent pas son choix d'un type d'école ou que les croyances et pratiques religieuses des parents n'influencent pas leurs choix quant au modèle d'éducation qu'ils préconisent pour leurs enfants. Et, bien entendu, on a une foule d'exemples de non-pratiquants, d'agnostiques, d'athées qui désirent que leurs enfants aillent à une école confessionnelle. De même que l'on a une foule d'exemples de parents qui, comme le veut l'expression, "retournent à la pratique " - pour un temps du moins - afin d'assurer à leurs enfants les pleins effets du type confessionnel d'éducation qu'ils ont choisi.

Cette inversion dans le mode de justification du caractère confessionnel des écoles est généralement expliquée de la façon suivante qui en montre l'importance pour notre propos: les gens, dirat-on, continuent de vouloir une école et un enseignement confessionnels parce qu'ils veulent donner une formation morale à leurs enfants; parce qu'en définitive, ils réduisent la religion à la morale. Or, dans le contexte confessionnaliste, cela est sûrement une excellente façon de désigner une morale sans fondement. Mais dans le contexte du monopole confessionnaliste, il devient nécessaire sinon de le préconiser, du moins de l'admettre comme mode institutionnel nécessaire au maintien du cadre actuel.'

Troisième remarque critique: donner à la conception de l'enseignement de la morale des principes théoriques qui consistent à articuler l'absence de fondement, c'est raisonner au sein d'une

5. De ce point de vue, on pourrait dire que graduellement le modèle confessionnel catholique épouse le modèle confessionel protestant. Le formalisme du premier tend à se rapprocher du nominalisme du second. Comme on le sait. pour des raisons historiques et autres, le modèle confessionnel protestant a de longue date fait place au pluralisme de sa clientèle. On dit qu'en réalité. depuis quelques années, la majorité des écoles protestantes du Québec nofoffait aucun enseignement religieux et moral. 
problématique rendue nécessaire par le monopole confessionnaliste pour qui il s'agit justement de trouver les cadres théoriques propres à cette dissociation par laquelle sont vécues la morale et la religion. Dans la mesure où le maintien du monopole confessionnaliste repose très précisément sur ce fait que "la religion n'est plus ce qu'elle était " tout en demeurant la seule possibilité structurale en matière de morale, il est important - et cela se fait par le biais de la critique des fondements - de maintenir le débat au niveau où justement on abandonne du même coup et fondement religieux et fondement tout court, et fondement et justification. Il est aussi impérieux de maintenir le débat dans le cadre formel dont je parlais tout à l'heure. En l'espèce, il est impérieux de diluer les conflits éthiques comme autant de conflits de toutes façons insolubles, aux enjeux de toutes façons provisoires et dans lesquels seuls des fanatiques - que ne sont plus les confessionnalistes - pourraient avoir quelque envie de se lancer. On pense trop qu'il faut s'opposer à ce qui est en grande partie sinon en totalité révolu: l'absolutisme éthique. On ne pense pas assez que le monopole confessionnaliste québécois perdure comme projet moral en faisant justement sien l'absence de fondement.

\section{LES SYMBOLES ÉTHIQUES}

Un dernier point. Il est maintenant admis que l'acceptation de l'école confessionnelle par les Québécois repose sur de multiples formes de raisons. Je parle ici d'acceptation plutôt que de " préférence ", comme on aime le dire sans avoir jamais sérieusement posé la question aux gens par le biais d'un recensement, comme l'avait recommandé le Rapport Parent. À ce sujet, il est intéressant de noter comment on utilise les divers sondages récents. On insiste sur le fait que la majorité des parents opte pour l'école confessionnelle, mettant en veilleuse la majorité de répondants pour qui la typologie actuelle des écoles devrait être modifiée. Ces derniers chiffres, on les noie en général en considérant le nombre infime des exemptés, mais sans trop insister sur le fait que le monopole confessionnaliste restreint la possibilité de leur augmentation ainsi que de nouvelles formules'.

o. À ce sujet, voir l'étude de Pierre Bouchard et Édouard Cloutier, Les attentes des parents vis-àias lácolle. Centre de sondage de l'Lniversité de Montréal, 1976, et l'étude dirigée par Normand Wener, Les aspirations des parents de l'fle de llontráal face a la religion et l'école, 
Mais pour revenir aux raisons qui militent en faveur de l'enseignement confessionnel, on admettra relativement facilement que ces raisons sont fréquemment a-religieuses, pour ne pas parler des raisons a-confessionnelles. Ainsi, on admettra que des raisons d'ordre psychologique (comme l'habitude), sociologique (comme le conformisme), éducative (comme l'adhésion au concept d'éducation intégrale), morale (comme le souci de donner des principes solides), juridique (comme le souci d'appliquer la loi et les règlements), historique (comme le renvoi à la tradition), social (comme la crainte du désordre), politique (comme la défense de structures linguistiques) puissent s'ajouter à des raisons d'ordre religieux (comme de prolonger l'engagement du baptême) ou même les remplacer. Mais il sera dit que les Québécois veulent, peut-être pour de mauvaises raisons, des écoles et un système confessionnels.

()r, on n'a pas suffisamment remarqué que de l'ensemble de ces raisons découle l'introduction tout à fait explicite du caractère historique des symboles éthiques. On ne dira plus, aujourd'hui, que cest " mal " de vouloir un enseignement confessionnel pour des raisons non religieuses ou pour des raisons qui n'ont rien à voir avec la morale. On ne le dira pas, parce que justement le monopole confessionnaliste assume le caractère ni religieux, ni " naturaliste" des symboles éthiques contemporains, voyant là du reste une preuve sinon de vertu, du moins de modernisme et en tout état de cause une condition impérieuse à son maintien comme symbole éthique.

Quatrieme remarque critique : poser comme principe théorique de l'enseignement de la morale l'évolution historique et con-

Conseil soblaire de lîte de Montréal, 1975. Voir aussi Jean-Marie Mathieu et Edmund J.

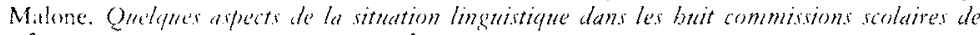

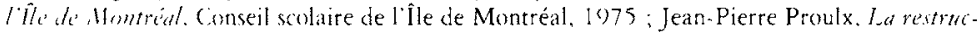

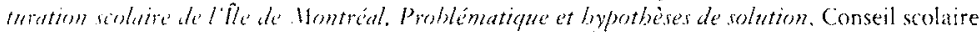

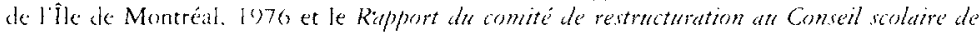
l'he de Hontridt. 1976.

A propes des exemptions, un droit reconnu en 1967 et intégré aux articles 14 et 16 des nouveaux règlenents (197-1) du Comité cathohique du Conseil supérieur de léducation, notons la création récente d'une association nommée AQADl:R (Association Québécoise pour I'Appli(ation du Droit a I'Exemption de l'Enseignement Religieux) dont l'un des principaux objectifs est de faite commaitre lexistence de ce droit.

7. Cela du reste ne va pas sans difficulté. Voir à ce sujet ma critique des principes théoriques présiatant i la formulation diune nouvelle approche de la confessionnalité catholique dans le do-

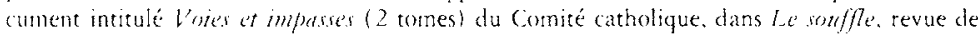

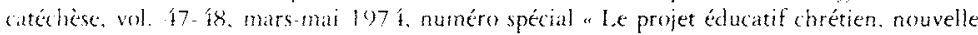
approhe de la confessionnalité ". Mon article s"intitule "Iln dépassement manqué " pp. 86 . [103. 
textuelle des valeurs, de leur perception, de leur symbole, de leur expression, -.- comme dans un enseignement, - c'est poser le principe même sur lequel s'appuie le monopole confessionnaliste dans la lente évolution qui l'a conduit au formalisme. Je dis que ce principe "photographie " cette situation plutôt qu'il ne l'éclaire. A tout le moins, il me paraît insuffisant en lui-même et tel quel pour faire avancer le débat, le monopole confessionnaliste perdurant par sa prise en charge même du caractère provisoire et du reste hétérogène des mobiles et éléments éthiques qu'il contient.

\section{CONCILISION}

Pour conclure ce commentaire, je dirai que mon soupçon concernant l'insuffisance des propositions défendues au cours du panel provient de ce que jappellerai "enfoncer des évidences". J'ai peur que l'on ait à l'esprit une image tout à fait révolue des enjeux, tels l'absolutisme, l'endoctrinement, l'intransigeance, la prédication, etc. Je veux dire une image révolue des enjeux théoriques prioritaires. la possibilité par exemple d'endoctrinement demeurant un danger en permanence. Car même dans le cas de ceux que l'on appelle les "irréductibles" - ceux qui reprochent aux écoles actuelles de n'être pas ou de n'être pas suffisamment confessionnelles, préconisant un retour au système d'avant les années soixante

il faut bien voir que leur critique n'opère plus au niveau où elle opérait jadis. Pour les "irréductibles", il s'agit moins d'assurer le monopole que de lui donner un contenu, quitte à le rompre d'ailleurs. Leur critique, en définitive, renvoie au problème fondamental de savoir si pour parler de morale et être critique, il suffit de refuser de s'engager dans tout contenu éthique déterminé et de refuser toute démarche de justification du contenu. De ce point de vue. le discours des "naturalistes" consiste moins à proposer quoi que ce soit qu'à affirmer par le biais de la morale naturelle, c'est-àdire par le biais de la soustraction des coefficients confessionnels, l'importance de dire quelque chose en éthique.

En d'autres termes, en m'interrogeant sur la question de savoir jusqu à quel point il y avait désaccord fondamental entre les panélistes, j'en suis venue à la conclusion qu'à beaucoup d'égards, ils opéraient tous à l'intérieur d'une problématique rendue nécessaire par le monopole confessionnaliste, d'où la difficulté de cerner la nature et la portée des désaccords dont on sentait bien par 
ailleurs l'existence. Ma suggestion est la suivante : en mettant en veilleuse les éléments structurants du contexte de la question qu'ils posent, tout en posant des principes dont tout le monde admettra qu'ils peuvent être interprétés de diverses façons, les panélistes ont tous photographié, à un moment ou à un autre, divers problèmes posés par le monopole confessionnaliste; alors qu'à mon avis il faut se situer critiquement face à ce dernier pour pouvoir formuler des principes d'orientation qui l'éclaireraient, y inclus des principes théoriques. Faute d'une mise en contexte, le sentiment que l'on a des différences entre les participants risque de demeurer au niveau des intuitions.

Université McGill 